\title{
Evaluating the Outcome of Two Different Regimes in Adhesive Capsulitis: A Prospective Clinical Study
}

\author{
Rashid Anjum $^{\mathrm{a}}$ Jatin Aggarwal ${ }^{\mathrm{b}}$ Rakesh Gautam ${ }^{\mathrm{b}}$ Subodh Pathak ${ }^{\mathrm{b}}$ \\ Aryan Sharmab \\ ${ }^{a}$ ASCOMS \& Hospital, Jammu, India; ${ }^{b}$ MM Institute of Medical Sciences and Research, Ambala, India
}

\section{Significance of the Study}

- Idiopathic adhesive capsulitis is a painful disorder affecting up to $5 \%$ of the general population.

- The variety of treatment modalities employed speaks for the fact that we still know little about the inciting etiology, pathogenesis, and response to treatment.

- Our study concludes that physiotherapy plus intra-articular steroid injection is more effective compared to physiotherapy alone in pain control and improvement in range of motion at short-term follow-up.

- More high-quality research with longer follow-up is required to establish the best modality for the management of idiopathic adhesive capsulitis.

\section{Keywords}

Adhesive capsulitis · Physiotherapy · Intra-articular steroid injection

\begin{abstract}
Objective: Adhesive capsulitis or frozen shoulder is a painful condition affecting up to $5 \%$ of the general population. We conducted this study with the aim of evaluating the results of physiotherapy plus intra-articular methylprednisolone injection versus physiotherapy alone in idiopathic frozen shoulder. Methods: This prospective clinical study was conducted in a tertiary care center between August 2016 and August 2018. Patients who were diagnosed with idiopathic frozen shoulder were included in the study, and each patient was randomly allocated to one of two groups: physiotherapy
\end{abstract}

\begin{tabular}{ll}
\hline KARGER & $\begin{array}{l}\text { () } 2019 \text { The Author(s) Karger } \\
\text { Published by S. Karger AG, Basel Open access }\end{array}$ \\
karger@karger.com & This is an Open Access article licensed under the Creative Commons \\
www.karger.com/mpp & $\begin{array}{l}\text { Attribution-NonCommercial-4.0 International License (CC BY-NC) } \\
\text { (http://www.karger.com/Services/OpenAccessLicense), applicable to } \\
\text { the online version of the article only. Usage and distribution for com- } \\
\text { mercial purposes requires written permission. }\end{array}$
\end{tabular}

alone (group A) and physiotherapy plus intra-articular steroid injection (group B). Results: A total of 52 cases diagnosed with idiopathic frozen shoulder were included and treated with the two modalities. There was a significant improvement in group B compared to group A at 6 weeks and 3 months in the range of flexion, abduction, and external rotation. The Shoulder Pain and Disability Index showed improvement in both pain and disability score in group $B$ compared to group $A$, and improvement was significant at 6 weeks and 3 months. Conclusion: The results demonstrate the advantages of physiotherapy plus intra-articular steroid injection in idiopathic frozen shoulder. The predictability of results with physiotherapy plus intra-articular steroid injection in selected patients is excellent, and it is a better modality of treatment compared to physiotherapy alone.

(c) 2019 The Author(s)

Published by S. Karger AG, Basel

Dr. Rashid Anjum, Assistant Professor Orthopaedics

A-4 Residential Complex ASCOMS

Jammu 180017 (India)

E-Mail raashidanjum@gmail.com 


\section{Introduction}

Stiffness of the shoulder is a symptom due to interlinked pathologies whose etiologies are complex and multifactorial. Treatment of these conditions is empirical and multifactorial. Adhesive capsulitis is a painful disorder that affects up to $4-5 \%$ of the general population [ 1 , 2]. Frozen shoulder can be primary (also called idiopathic), which means that no identifiable cause can be found, or secondary when the cause is known, such as any systemic disease like diabetes or trauma [3]. In 1934, Codman [3] introduced the term "frozen shoulder" for patients who present with pain in the shoulder that is insidious in onset and associated with shoulder stiffness that results in diminished range of motion (ROM; primarily flexion and external rotation of the shoulder) [4]. The patients usually present after the fifth decade of life and the disorder is more common in women; however, Bunker suggested that the incidence is equal among males and females $[2,5]$. In up to $17 \%$ of patients, both shoulder joints are affected. The other shoulder joint is affected within 5 years after the first has resolved [2]. Primary frozen shoulder develops without any specific precipitating factor or cause. It involves a chronic inflammatory process in which there is proliferation of fibroblasts due to a hyperactive immune response. Secondary frozen shoulder occurs due to a shoulder trauma or surgery and may also occur due to any systemic illness or conditions such as diabetes, cardiac problems, and rotator cuff injury that may increase recovery time.

Primary frozen shoulder has three clinical phases: (1) The painful phase. Patients have mild to moderate dull aching pain even at rest similar to impingement syndrome and rotator cuff tendinosis. It occurs due to synovitis. This phase lasts for up to 9 months. (2) The stiffening or frozen phase. It is a continuum of the first phase in which pain is relieved, but movements are restricted due to capsular scarring. Poor scapulohumeral rhythm is demonstrable. It lasts for up to 12 months. (3) The thawing phase. This is characterized by slow and steady recovery of shoulder function. Capsular remodeling occurs and the pain resolves, but the functional residue persists to some extent.

The treatment of frozen shoulder is controversial and various modalities are en vogue. Certain authors have described "benign neglect," which is based on the finding that the natural course of the disease is self-limiting and pain resolves by itself after some period of time (1-3 years) when no medical intervention has been done. Various modalities have been prescribed to improve pain and shoulder movement and include physical therapy, oral corticosteroids, NSAIDs, injections (corticosteroid, local anesthetic, sodium hyaluronate, calcitonin), articular or arthrography distension, manipulation under anesthesia, arthroscopic and open surgery, or a combination of these. Conservative management is done so as to improve the pain and shoulder function by stretching exercises and to provide symptomatic relief [3-6]. Corticosteroid injections into the glenohumeral and the subacromial joint have been found to decrease the inflammation of synovial membrane and fibrosis of the capsule and to improve shoulder movement and pain [7].

We conducted this study with the aim of evaluating and comparing the outcome of physiotherapy and corticosteroid injection with physiotherapy alone in terms of reduction in pain and improvement in ROM.

\section{Materials and Methods}

This randomized prospective study was conducted in a tertiary care center between August 2016 and August 2018. Patients with diagnosed idiopathic frozen shoulder of $<6$ months' duration who consented to take part in the study were included. Each patient was randomly allocated to one of two groups: physiotherapy alone (group A) and physiotherapy plus intra-articular steroid injection (group B). A total of 52 patients aged between 18 and 65 years participated in the study. The criteria for inclusion were age between 18 and 65 years, nontraumatic stiff shoulder, nondiabetic state, and restriction of active and passive motion $>30^{\circ}$ in two or more planes. The criteria for exclusion were systemic inflammatory joint disease, skeletally immature patients, contraindications to joint distension including allergy to local anesthetic, and shoulder abnormality detected on plain X-ray. The diagnosis of idiopathic frozen shoulder was made on the basis of history, physical examination, and clinicoradiological examination. Our study included patients of both sexes who were skeletally mature and had clinical signs and symptoms of primary frozen shoulder. ROM was evaluated at the shoulder joint, i.e., active and passive shoulder flexion, abduction, as well as internal and external rotation with the patients standing or sitting. X-ray or MRI of the shoulder joint was carried out in some patients to exclude conditions other than frozen shoulder, but were not done routinely as frozen shoulder is a clinical diagnosis. Glenohumeral osteoarthritis and neoplasm were excluded by radiographs. Pain related to acromioclavicular joint or biceps was ruled out based on lack of tenderness with palpation of these structures. Those patients with confirmed idiopathic adhesive capsulitis were taken up for a trial of physiotherapy (group A) or physiotherapy plus intra-articular steroid injection (group B). Group A patients were assigned to the following physiotherapy protocol: capsular stretching (from day 1), modified sleeper's stretch and cross-body stretch with breathing, ROM exercises (from day 1), pendulum exercises, and active assisted exercises (wand exercises). Isometric strengthening exercises were started on day 1 whereas isotonic concentric exercises were started on day 14. A moist heat pack was applied before starting exercises 
Table 1. Demographic details of patients among the two groups

\begin{tabular}{lll}
\hline Variable & Group A & Group B \\
\hline Mean age, years & 41.12 & 44.46 \\
Female & $15(58 \%)$ & $17(65 \%)$ \\
Male & $11(42 \%)$ & $9(35 \%)$ \\
Right shoulder affected & $10(38 \%)$ & $10(38 \%)$ \\
Left shoulder affected & $14(54 \%)$ & $13(50 \%)$ \\
Bilateral & 2 & 3 \\
\hline
\end{tabular}

Group A, physiotherapy alone; group B, physiotherapy plus intra-articular steroid injection.

each time. Group B patients, in addition to the same protocol of physiotherapy as in group A, were given an intra-articular methylprednisolone injection ( $80 \mathrm{mg}$ methylprednisolone mixed with $0.5 \%$ bupivacaine to make a 10 - $\mathrm{mL}$ solution) and were given analgesics only during episodes of severe pain. We performed all intraarticular steroid injections under aseptic conditions in the setting of a minor operation theater with the patient in a sitting position through posterior approach after palpating and marking landmarks. After receiving the injection, patients were advised to undergo exercises immediately as per protocol and were followed up subsequently at 2 weeks, 6 weeks, and 3 months. Shoulder function was evaluated and documented at the time of presentation and at each subsequent visit by Shoulder Pain and Disability Index (SPADI) and ROM at forward flexion, internal rotation, external rotation, and abduction. Similarly, improvement in pain score was assessed at subsequent visits by using a visual analog scale (VAS). Flexion and abduction of the shoulder was done by measurement of the angle formed between the arm and thorax. External rotation was measured with the arm in adduction and the elbow at the side and flexed to $90^{\circ}$. Internal rotation of the shoulder was done by the arm behind the back and assessment was determined by the vertebral level that could be reached by the tip of the thumb at the highest spinous process.

\section{Results}

Our study included 52 patients diagnosed with idiopathic adhesive capsulitis of the shoulder. All patients were right hand dominant. The mean age of the patients in group A was 41.12 years and that in group B was 44.46 years. There were 15 (58\%) females in group A compared to 17 (65\%) in group B (Table 1). The left side was more commonly involved compared to the right side in both groups. However, none of these parameters reached statistical significance. Abduction and external rotation demonstrated the most significant and rapid recovery, with internal rotation being the most difficult to recover among both groups with greatest improvement in group B. Flexion, abduction, and external rotation demonstrat-

Finding the Best Way out in Frozen Shoulder ed significant recovery in group B in comparison to group A $(p<0.05)$ at 6 weeks and 3 months. There was no significant improvement in flexion, abduction, and external rotation at 2 weeks. The SPADI showed significant improvement in both pain and disability score in group B $(p<0.05)$ at 6 weeks and 3 months. Most of the patients were able to sleep on the affected side without disturbing their sleep and were able to return to their normal daily routine activities. The VAS also showed significant improvement in group $\mathrm{B}(p<0.05)$ at 6 weeks and 3 months. The mean improvement in group $B$ in the range of internal rotation at 3 months was 9 spinous process in comparison to group A having improvement of 7 spinous process. There was a significant improvement in abduction, flexion, and external rotation at 3 months. The mean improvement in the pain score in this group at 3 months was $45.61 \%$, with a mean pain score of $66.23 \%$ on day 0 , $52.00 \%$ at 2 weeks, $37.38 \%$ at 6 weeks, and $20.62 \%$ at 3 months. The mean improvement in the disability score in this group at 3 months was $42.30 \%$, with a mean disability score of $60.14 \%$ on day $0,47.64 \%$ at 2 weeks, $30.29 \%$ at 6 weeks, and $17.84 \%$ at 3 months. The mean improvement in the SPADI score in this group at 3 months was $43.58 \%$, with a mean SPADI score of $62.49 \%$ on day 0 , $49.32 \%$ at 2 weeks, $33.02 \%$ at 6 weeks, and $18.91 \%$ at 3 months. There was a significant improvement in pain score, disability score, and SPADI score at 3 months. ROM, rotator cuff power, and functional outcome by SPADI and VAS at inclusion, 2 weeks, 6 weeks, and 3 months are shown in Table 2.

\section{Discussion}

Frozen shoulder remains an enigma and is a difficult problem to manage. The variety of treatment modalities employed speaks to the fact that little is known about the inciting etiology, pathogenesis, natural history, and response to treatment. Treatment with physiotherapy, deep heat, and stretching exercises improves the symptoms of frozen shoulder as these leads to stretching of the shoulder capsule. In primary frozen shoulder, the onset of symptoms is spontaneous, with no recognizable precipitating event. Most patients correlate the onset of symptoms with some trivial trauma. Similar findings have been reported in the literature. In our series, patients presented to us at an average of 7 weeks after the onset of symptoms. The age of the patients with idiopathic frozen shoulder ranged from 25 to 55 years in group A (physiotherapy) and from 22 to 68 years in group B (physiotherapy plus 
Table 2. Comparative analysis of SPADI, pain score, and ROM between groups $\mathrm{A}$ and $\mathrm{B}$

\begin{tabular}{|c|c|c|c|}
\hline Variable & Group A & Group B & $p$ value \\
\hline \multicolumn{4}{|l|}{ SPADI, \% } \\
\hline At inclusion & $62.28(8.298)$ & $62.49(8.758)$ & 0.930 \\
\hline 2 weeks & $50.33(6.685)$ & $49.32(6.062)$ & 0.572 \\
\hline 6 weeks & $38.11(6.685)$ & $33.02(4.975)$ & 0.000 \\
\hline 3 months & $24.41(5.722)$ & $18.91(3.995)$ & 0.000 \\
\hline \multicolumn{4}{|l|}{ Pain score, $\%$} \\
\hline At inclusion & $67.46(11.136)$ & $66.23(10.915)$ & 0.689 \\
\hline 2 weeks & $55.92(7.579)$ & $52.00(10.613)$ & 0.131 \\
\hline 6 weeks & $42.15(5.985)$ & $37.38(9.029)$ & 0.029 \\
\hline 3 months & $27.00(6.603)$ & $20.62(6.034)$ & 0.000 \\
\hline \multicolumn{4}{|c|}{ Disability score, $\%$} \\
\hline At inclusion & $59.04(9.594)$ & $60.14(13.552)$ & 0.735 \\
\hline 2 weeks & $46.83(8.210)$ & $47.64(10.743)$ & 0.759 \\
\hline 6 weeks & $35.58(7.204)$ & $30.29(6.258)$ & 0.006 \\
\hline 3 months & $22.79(7.602)$ & $17.84(5.674)$ & 0.010 \\
\hline \multicolumn{4}{|l|}{ VAS score } \\
\hline At inclusion & $5.69(1.289)$ & $5.92(1.262)$ & 0.517 \\
\hline 2 weeks & $4.00(1.070)$ & $4.23(1.070)$ & 0.429 \\
\hline 6 weeks & $2.69(0.884)$ & $2.19(0.567)$ & 0.018 \\
\hline 3 months & $1.77(0.710)$ & $1.15(0.464)$ & 0.000 \\
\hline \multicolumn{4}{|c|}{ Supraspinatus power } \\
\hline At inclusion & $4.42(0.703)$ & $4.54(0.706)$ & 0.557 \\
\hline 3 months & $4.73(0.452)$ & $4.85(0.368)$ & 0.317 \\
\hline \multicolumn{4}{|c|}{ Infraspinatus power } \\
\hline At inclusion & $4.62(0.496)$ & $4.73(0.452)$ & 0.385 \\
\hline 3 months & $5.00(0.000)$ & $5.00(0.000)$ & ND \\
\hline \multicolumn{4}{|c|}{ Subscapularis power } \\
\hline At inclusion & $4.69(0.471)$ & $4.73(0.452)$ & 0.765 \\
\hline 3 months & $4.92(0.272)$ & $5.00(0.000)$ & 0.155 \\
\hline \multicolumn{4}{|l|}{ Flexion } \\
\hline At inclusion & $126.92(24.782)$ & $123.65(28.76)$ & 0.662 \\
\hline 2 weeks & $138.08(24.169)$ & $137.88(23.45)$ & 0.976 \\
\hline 6 weeks & $148.08(15.942)$ & $156.15(11.42)$ & 0.040 \\
\hline 3 months & $159.04(11.834)$ & $169.62(6.917)$ & 0.000 \\
\hline \multicolumn{4}{|l|}{ External rotation } \\
\hline At inclusion & $33.46(15.347)$ & $34.81(14.525)$ & 0.746 \\
\hline 2 weeks & $40.58(13.441)$ & $47.31(11.334)$ & 0.056 \\
\hline 6 weeks & $47.88(18.394)$ & $59.23(15.277)$ & 0.019 \\
\hline 3 months & $53.46(17.480)$ & $66.92(13.717)$ & 0.003 \\
\hline \multicolumn{4}{|l|}{ Abduction } \\
\hline At inclusion & $72.31(26.124)$ & $80.77(30.584)$ & 0.288 \\
\hline 2 weeks & $82.31(24.667)$ & $91.35(28.339)$ & 0.225 \\
\hline 6 weeks & $93.08(26.156)$ & $109.62(27.565)$ & 0.031 \\
\hline 3 months & $114.04(20.737)$ & $134.81(21.931)$ & 0.001 \\
\hline \multicolumn{4}{|c|}{ Hand behind back } \\
\hline At inclusion & S3 & S3 & \\
\hline 2 weeks & L5 & L5 & \\
\hline 6 weeks & $\mathrm{L} 2$ & L1 & \\
\hline 3 months & $\mathrm{L} 1$ & D11 & \\
\hline
\end{tabular}

Values are presented as mean \pm standard deviation. Group A, physiotherapy alone; group $\mathrm{B}$, physiotherapy plus intra-articular steroid injection; ND, no difference; ROM, range of motion; SPADI, Shoulder Pain and Disability Index; VAS, visual analog scale. intra-articular steroid injection) in our series. Codman [3] and Lundberg [8] showed that the disease occurred in males at a relatively younger age than in females. Codman [3] and Lippman [9] found adhesive capsulitis to be more common in females in their study. The left side was reported to be more commonly involved compared to the right side [10]. We observed similar results: all patients in our series were right hand dominant, but the left side was seen to be more commonly involved compared to the right side in both groups; however, the difference did not reach statistical significance. Two patients in group A had bilateral involvement and 3 patients in group $\mathrm{B}$ had bilateral involvement. No correlation was observed between the duration of presenting symptoms and the Oxford Shoulder Score at the initial follow-up or at long-term follow-up [11]. However, Flannery et al. reported 145 shoulders treated with manipulation under anesthesia following 3 months of unsuccessful conservative treatment $[12,13]$. They found significantly better Oxford Shoulder Score and ROM in patients who presented early in the course of the disease. In our series, the mean improvement in the flexion in group A at 3 months was $32.12^{\circ}$, with a mean $\mathrm{ROM}$ of $126.92^{\circ}$ on day $0,138.08^{\circ}$ at 2 weeks, $148.08^{\circ}$ at 6 weeks, and $159.04^{\circ}$ at 3 months. Compared to this the mean improvement in the flexion in group B at 3 months was $45.97^{\circ}$, with a mean ROM of $123.65^{\circ}$ on day $0,137.88^{\circ}$ at 2 weeks, $156.15^{\circ}$ at 6 weeks, and $169.62^{\circ}$ at 3 months. There was a significant improvement at 3 months.

Although frozen shoulder is believed to be a self-limiting condition [3] that can be treated frequently with physical therapy, it is difficult to identify a precise endpoint for the resolution of symptoms or restriction of motion and a return to a normal state. Many studies have shown long-term functional disability [14]. Rizk et al. reported that of patients who received physical therapy along with other modalities, only $60 \%$ achieved the ability to sleep pain-free after 5 months [15]. Little long-term advantage in using intra-articular steroid injections, mobilizations, ice therapy, or no treatment has been reported, but steroid injections were shown to benefit pain and ROM in the early stages [16]. A 50\% improvement in pain scores after intra-articular injections was noted, but with only a $13 \%$ increase in ROM [11]. In our series, there was significant improvement in the range of external rotation at 3 months. Similarly, there was a significant improvement in the range of abduction at 3 months in both groups, but the final results of steroid injection and physiotherapy were better than those of physiotherapy alone. 
Carette et al. [7] demonstrated a statistically significant improvement after treatment with corticosteroid injection plus exercise versus exercise alone or placebo treatment. Another study by Marx et al. [13] showed that intra-articular injection in the early stages of adhesive capsulitis allows better and quicker recovery. In our series, the mean improvement in the pain score in group A at 3 months was $40.46 \%$ compared to $45.61 \%$ in group $\mathrm{B}$.

In a randomized controlled trial (RCT) comparing the effectiveness of two different physiotherapy regimens in 77 patients, Diercks and Stevens [17] showed that intensive physical rehabilitation can be counterproductive, comparing patients in a first group performing passive stretching and mobilization beyond pain limits with a second group of patients performing active and actively assisted exercises within pain limits (supervised neglect). At 2 years only $63 \%$ of the first group and $89 \%$ of the second group had good shoulder function. A systematic review of RCTs and quasi-randomized studies studying the effect of manual therapy and its various combinations in the management of frozen shoulder concluded that physiotherapy alone may not be as effective as steroid injection in the short term and highlighted the need for high-quality RCTs to establish the pros and cons of manual therapy and exercise interventions compared with placebo, no intervention, and active interventions like steroid injection [18]. A systematic review comparing the effectiveness of conservative and surgical interventions in adhesive capsulitis showed strong evidence in favor of the effectiveness of steroid injection and laser therapy at short term and moderate evidence supporting the same at mid term. Moderate evidence supporting mobilization techniques and active physiotherapy at short- and longterm follow-up was reported. The authors further highlighted the need for further research and RCTs in this area [19]. Although many studies showed significant early benefit of intra-articular injections on pain and shoulder ROM, no differences were found after 3 months between the various treatments. They may be more effective in the early inflammatory stage without substantial capsular contracture, but this finding has yet to be proven with higher-level studies $[20,21]$. In our study, the combination of steroid injection with physiotherapy appeared to be more effective than physiotherapy alone in the recovery of ROM. D'Orsi et al. [22] reported similar outcomes in their study. Sodium hyaluronate injection into the shoulder joint is a relatively new intervention for the management of adhesive capsulitis. Hyaluronate is a major component of connective tissue and has metabolic effects on articular cartilage, synovial tissue, and synovial fluid [23]. Hyaluronic acid injections have been shown to be beneficial for persistent shoulder pain, especially when the main reason is osteoarthritis of the glenohumeral joint [24, 25]. Hyaluronate injection into the glenohumeral joint appears to be a safe and effective procedure improving pain and ROM at short-term follow-up in patients with adhesive capsulitis of the shoulder [26]. However, we have no practical experience with hyaluronate injections in idiopathic adhesive capsulitis. In our study, the majority of patients in group B had significant improvement in pain and ROM, indicating that good results are obtained if intervention is done in phase 1 of adhesive capsulitis. Although we obtained satisfactory results, longer duration of follow-up and refinement of the procedure with the use of fluoroscopy or ultrasound might have further improved the results.

\section{Conclusion}

Though the sample size in the present study was small, the results show the efficacy of the combined approach in the management of idiopathic adhesive capsulitis. The predictability of results with physiotherapy plus intra-articular steroid injection in selected patients is more effective compared to physiotherapy alone for pain control and improvement in ROM at short-term follow-up. However, there is a need for further research with longer follow-up to formulate the best management protocol for idiopathic adhesive capsulitis.

\section{Statement of Ethics}

Ethical clearance was obtained from the Institutional Review Board of Maharishi Markandeshwar Institute of Medical Sciences and Research, Mullana, Haryana, India.

\section{Disclosure Statement}

The authors have no conflict of interest to disclose; no financial assistance was taken for any part of this study. 


\section{References}

1 Shah N, Lewis M. Shoulder adhesive capsulitis: systematic review of randomised trials using multiple corticosteroid injections. $\mathrm{Br} \mathrm{J}$ Gen Pract. 2007 Aug;57(541):662-7.

2 Bunker T. Time for a new name for frozen shoulder - contracture of the shoulder. Shoulder Elbow. 2009;1(1):4-9.

3 Codman EA. The Shoulder. Boston: Thomas Told; 1934.

4 Dias R, Cutts S, Massoud S. Frozen shoulder. BMJ. 2005 Dec;331(7530):1453-6.

5 Ombregt L, Bisschop P, TerVeer HJ. A System of Orthopaedic Medicine. 2nd ed. London: Churchill Livingstone; 2003.

6 Croft P. Soft tissue rheumatism. In: Silman AJ, Hochber MS, editors. Epidemiology of the rheumatic diseases. Oxford: Oxford Medical Publications; 1993. p. 375-421.

7 Carette S, Moffet H, Tardif J, Bessette L, Morin $\mathrm{F}$, Frémont $\mathrm{P}$, et al. Intraarticular corticosteroids, supervised physiotherapy, or a combination of the two in the treatment of adhesive capsulitis of the shoulder: a placebocontrolled trial. Arthritis Rheum. 2003 Mar 48(3):829-38.

8 Lundberg BJ. The frozen shoulder. Clinical and radiographical observations. The effect of manipulation under general anesthesia. Structure and glycosaminoglycan content of the joint capsule. Local bone metabolism. Acta Orthop Scand Suppl. 1969;119:1-59.

9 Lippmann RK. Frozen shoulder; periarthritis; bicipital tenosynovitis. Arch Surg. 1943;47(3): 283-96.

10 Depalma AF. Loss of scapulohumeral motion (frozen shoulder). Ann Surg. 1952 Feb;135(2): 193-204.
11 Thomas WJ, Jenkins EF, Owen JM, Sangster MJ, Kirubanandan R, Beynon C, et al. Treatment of frozen shoulder by manipulation under anaesthetic and injection: does the timing of treatment affect the outcome? J Bone Joint Surg Br. 2011 Oct;93(10):1377-81.

12 Flannery O, Mullett $\mathrm{H}$, Colville J. Adhesive shoulder capsulitis: does the timing of manipulation influence outcome? Acta Orthop Belg. 2007 Feb;73(1):21-5.

13 Marx RG, Malizia RW, Kenter K, Wickiewicz TL, Hannafin JA. Intra-articular corticosteroid injection for the treatment of idiopathic adhesive capsulitis of the shoulder. HSS J. 2007 Sep;3(2):202-7.

14 Reeves B. Arthrography of the shoulder. J Bone Joint Surg Br. 1966 Aug;48(3):424-35.

15 Rizk TE, Christopher RP, Pinals RS, Higgins AC, Frix R. Adhesive capsulitis (frozen shoulder): a new approach to its management. Arch Phys Med Rehabil. 1983 Jan;64(1):29-33.

16 Bulgen DY, Binder AI, Hazleman BL, Dutton J, Roberts S. Frozen shoulder: prospective clinical study with an evaluation of three treatment regimens. Ann Rheum Dis. 1984 Jun;43(3):353-60.

17 Diercks RL, Stevens M. Gentle thawing of the frozen shoulder: a prospective study of supervised neglect versus intensive physical therapy in seventy-seven patients with frozen shoulder syndrome followed up for two years. J Shoulder Elbow Surg. 2004 Sep-Oct;13(5): 499-502.

18 Page MJ, Green S, Kramer S, Johnston RV, McBain B, Chau M, et al. Manual therapy and exercise for adhesive capsulitis (frozen shoulder). Cochrane Database Syst Rev. 2014 Aug; 8:CD011275.
19 Favejee MM, Huisstede BM, Koes BW. Frozen shoulder: the effectiveness of conservative and surgical interventions - systematic review. Br J Sports Med. 2011 Jan;45(1):49-56.

20 Tveitå EK, Tariq R, Sesseng S, Juel NG, BautzHolter E. Hydrodilatation, corticosteroids and adhesive capsulitis: a randomized controlled trial. BMC Musculoskelet Disord. 2008 Apr;9:53.

21 Griesser MJ, Harris JD, Campbell JE, Jones GL. Adhesive capsulitis of the shoulder: a systematic review of the effectiveness of intraarticular corticosteroid injections. J Bone Joint Surg Am. 2011 Sep;93(18):1727-33.

22 D’Orsi GM, Via AG, Frizziero A, Oliva F. Treatment of adhesive capsulitis: a review. Muscles Ligaments Tendons J. 2012 Sep;2(2): 70-8.

23 Moskowitz RW, Blaine TA. An overview of treatment options for persistent shoulder pain. Am J Orthop (Belle Mead NJ). 2005 Dec; 34(12 Suppl):10-5.

24 Blaine T, Moskowitz R, Udell J, Skyhar M, Levin R, Friedlander J, et al. Treatment of persistent shoulder pain with sodium hyaluronate: a randomized, controlled trial. A multicenter study. J Bone Joint Surg Am. 2008 May; 90(5):970-9.

25 Harris JD, Griesser MJ, Copelan A, Jones GL. Treatment of adhesive capsulitis with intraarticular hyaluronate: A systematic review. Int J Shoulder Surg. 2011 Apr;5(2):31-7.

26 Georgiannos D, Markopoulos G, Devetzi E, Bisbinas I. Adhesive Capsulitis of the Shoulder. Is there Consensus Regarding the Treatment? A Comprehensive Review. Open Orthop J. 2017 Feb;11(1):65-76. 